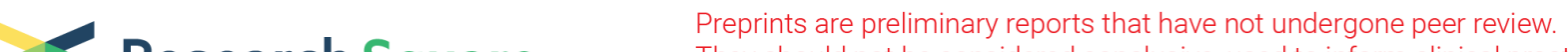 Research Square They should not be considered conclusive, used to inform clinical practice, or referenced by the media as validated information.
}

\section{Endoscopic Endoclip Papilloplasty \\ Preserves Sphincter of Oddi Function After Endoscopic Sphincterotomy in Porcine Models}

\section{YingChun Wang}

Peking University Third Hospital

Hong Chang

Peking University Third Hospital

YaoPeng Zhang

Peking University Third Hospital

Kun Wang

Peking university third Hospital

HeJun Zhang

Peking University Third Hospital

XiuE Yan

Peking University Third Hospital

LingYu Meng

Peking University Third Hospital

Wei Yao

Peking University Third Hospital

Ke Li

Peking University Third Hospital

YongHui Huang (D 15611908652@163.com )

Peking university third hospital https://orcid.org/0000-0002-0238-746X

\section{Research article}

Keywords: Endoscopic endoclip papilloplasty, Endoscopic sphincterotomy, Sphincter of Oddi dysfunction, recurrent choledocholithiasis, porcine model

Posted Date: May 7th, 2020

DOI: https://doi.org/10.21203/rs.3.rs-22693/v1

License: (c) (1) This work is licensed under a Creative Commons Attribution 4.0 International License.

Read Full License 


\section{Abstract \\ Background}

Endoscopic sphincterotomy (EST) can destroy sphincter of Oddi (SO) function and plays a role in recurrent choledocholithiasis and cholangitis development. The purpose of this study was to assess the feasibility of endoscopic endoclip papilloplasty (EEPP) in restoring SO function after EST.

\section{Methods}

Seven 26-week-old domestic pigs were divided into control and EEPP groups. Necropsy, hematoxylineosin staining, anti-a-smooth muscle actin (a-SMA) staining of papilla and sphincter of Oddi manometry (SOM) were conducted in animals at three independent time points.

\section{Results}

EST and EEPP were safely performed in all 7 pigs without serious adverse events. For primary outcome, compared to the controls, EEPP generated smaller dilation with less inflammation. Fibrous repair of the papilla was observed at 24 weeks after EEPP. For secondary outcome, in the control group, SO basal pressure ( $17.25 \pm 18.14$ to $5.50 \pm 0.71 \mathrm{mmHg}$ ), SO contraction amplitude ( $46.00 \pm 19.20$ to $34.50 \pm$ $48.79 \mathrm{mmHg})$, peak ( $4.50 \pm 4.04$ to $1.50 \pm 2.12)$ and frequency $(3.05 \pm 3.29$ to $1.41 \pm 2.19 / \mathrm{min})$ reduced after EST. Further reductions to almost 0 were observed 3 weeks later, including common bile duct pressure and SO contraction period. In contrast, in the EEPP group, these manometric data recovered to pre-EST levels, such as CBD pressure ( $11.5 \pm 7.31$ vs $11 \pm 2.16 \mathrm{mmHg})$, SO pressure (17.50 $\pm 17.75 \mathrm{vs}$ $18.20 \pm 21.39 \mathrm{mmHg})$ and SO contraction amplitude $(53.67 \pm 21.54$ vs $60.00 \pm 36.08 \mathrm{mmHg})$. However, no significant differences were observed by Student $t$-test.

\section{Conclusions}

In this porcine study, EEPP had accelerated and improved papillary healing after EST, and preserved SO function.

\section{Background}

Since its first application for bile stone extraction in 1974 [1, 2], endoscopic sphincterotomy (EST) has been widely practiced and become a standard therapy in managing choledocholithiasis. However, there are short term and late complications of EST, such as recurrent choledocholithiasis with an incidence of $8.9 \% \sim 12.3 \%[3-6]$. Biliary bacteria reflux, surgery or EST, abnormal biliary anatomy, periampullary duodenum diverticulum, and chronic inflammation were the well-established risk factors for recurrent choledocholithiasis [7]. Sphincter of Oddi (SO) maintains the pressure gradient between the common bile 
duct (CBD) and the duodenal lumen, preventing duodenal fluid and bacterial reflux into the bile duct[8]. Yang et.al have demonstrated that after EST, SO contraction amplitude and frequency reduced markedly, and bacterial reflux into the bile duct played a role in the development of recurrent choledocholithiasis [9]. Recently, we have reported a novel method to close patulous papilla after EST: endoscopic endoclip papilloplasty (EEPP) [10]. The purpose of this animal study was to assess the feasibility of EEPP in restoring SO function after EST and to demonstrate its histological and manometric evidence. The authors compared the histological and manometric changes of papilla after EST or EEPP in a porcine model.

\section{Methods}

All experimental procedures in this study were approved by the Ethical Committee of Laboratory Animals of Peking University Third Medical School.

Animal preparations

Seven 26-week-old domestic pigs (Bama Minipigs; Beiqijia meile farm, Beijing, China) were used. Randomly, animals were divided into 2 groups, 4 in EEPP group and 3 in control group. The pigs were daily visual inspected during an acclimatization period of 7 days and fasted for 24 hours before the procedures. These pigs had commercial semisolid swine feed (Beijing Keao Xieli Feed Co., Ltd., Beijing, China). All animals underwent general endotracheal anesthesia, induced with sodium pentobarbital and maintained with propofol. Base-line vital signs (pulse, respiratory rate, arterial blood pressure, $\mathrm{O}_{2}$ saturation) were monitored and recorded every 5 minutes during the procedure. Finally, all animals were sacrificed by overdose sodium pentobarbital intravenous injection as indicated below. The primary experimental outcome was histological changes after operations. The secondary experimental outcome was SO manometry changes 3 weeks after procedures.

\section{Study models (EEPP)}

Endoscopic retrograde cholangiography was performed using a standard duodenoscope (ED-450XT, Fujifilm Medical Systems; Tokyo, Japan). The EEPP procedure was performed as described previously[10, 11]. Selective wire-guided (Jagwire, Boston Scientific, MA, U.S) biliary cannulation was achieved using a standard sphincterotome (Autotome Rx, Boston Scientific). After a large size EST $(>1 \mathrm{~cm}$ ) and a plastic biliary stent (Advanix, Boston Scientific) placement. The patulous papilla was zipper closed using Sure clips (Rocc-D-26-195, Micro-Tech Co., Ltd., NanJing, China) in a vertically linearized manner (Figure 1). 
After successful wire-guided selective cannulation of bile duct, a manometric pressure recording catheter (Cook Medical. Indiana, U.S.) was placed into the CBD and confirmed on fluoroscopy. The external end of the pressure catheter was attached to an analyzing machine (PC-Polygraf HR, CTD. SYNECTICS, Sweden). Once deep cannulation was achieved, we first recorded the CBD pressure. Then the catheter was withdrawn across the sphincter at 1-2 mm intervals by standard station pull-through technique until a phasic high-pressure zone was located. Maintained the catheter at that place for at least 3-5 minutes, then the high pressure zone was recorded as the basal pressure of SO. Meanwhile, we recorded the amplitude, period, frequency and peaks of SO contractions. Then we pulled the pressure catheter into the duodenal lumen and recorded the duodenal pressure.

Hematoxylin-eosin and Immunohistochemistry staining

Three weeks after EST \pm EEPP procedure, 6 pigs were sacrificed by overdose sodium pentobarbital intravenous injection, including 3 in the control group and 3 in the EEPP group. The papilla was removed from euthanized animals for examination by staining with hematoxylin-eosin. Histological and pathological evaluations were performed by two experienced pathologists separately. Twenty-four weeks after EEPP procedure, the last pig was euthanized by overdose sodium pentobarbital intravenous injection. Besides hematoxylin-eosin staining, tissue sections from the removed papilla were stained with a mouse monoclonal anti-a-smooth muscle actin (a-SMA) antibody (Invitrogen, Thermo Fisher scientific, MA, U.S.) for 60 minutes.

Statistical analysis

Data obtained from 3 independent pigs were analyzed by Student $t$ test depending on the result of

Levine's test for equality of variance. Value of $p<0.05$ was considered to indicated statistically significant differences. All statistical analyses were performed using SPSS 25.0 (IBM Corp, Armonk, NY).

\section{Results}

The safety of EST+EEPP was comparable with stand-alone EST

EST and EEPP were safely achieved in both control $(n=3$, underwent large EST only) and EEPP $(n=4)$ pigs. No serious adverse event was observed, such as obvious immediately bleeding, perforation, irritability or death. The EEPP procedure time was about 40 to 80 minutes (Figure 1). 
Endoscopic examination was performed 3 weeks after EST/EEPP. The control group showed significant papillary patulous opening and deformation as compared to normal untreated papilla. In contrast, the EEPP group showed a smaller dilation and only mild deformation (Figure 2).

After endoscopic examination and SOM, animals were euthanized and examined for papilla histological changes 3 weeks after procedures. For primary outcome, the control group displayed severe inflammatory responses, including abundant neutrophil and macrophage infiltrations, hyperphlogosis, poor angiogenesis, large inflammatory area, severe hemorrhage, irregular wound surface and suboptimal renovation (Figure 3, A-C). In contrast, the EEPP group demonstrated mild inflammatory responses, such as subtle neutrophil and macrophage infiltrations, good angiogenesis, small inflammatory area, mild hemorrhage, tidy wound surface and optimal renovation (Figure 3, D-F). Furthermore, one pig in EEPP group was euthanized and investigated for papilla histological changes until 24 weeks after procedure. The hematoxylin-eosin staining showed repaired connective tissue in the wound surface, along with hyperplastic fibrous connective tissue and angiogenesis. By anti-a-SMA staining showed myofibroblasts between the fibrous connective tissue and vessels. All these findings indicate a fibrous repair of the papilla after EEPP.

EEPP restored the SO function after large EST

SOM was conducted at three time points: 1) immediately after CBD cannulation, before EST/EEPP procedures, 2) after large EST, and 3) three weeks after EST/EEPP. For secondary outcome, in control group, SO pressure( $17.25 \pm 18.14$ to $5.50 \pm 0.71 \mathrm{mmHg})$, SO contraction amplitude( $46.00 \pm 19.20$ to $34.50 \pm 48.79 \mathrm{mmHg})$, SO contraction peaks $(4.50 \pm 4.04$ to $1.50 \pm 2.12)$ and SO contraction frequency $(3.05 \pm 3.29$ to $1.41 \pm 2.19 / \mathrm{min})$ reduced after EST. Similar results were observed in EEPP group, including CBD pressure $(11.00 \pm 2.16$ to $8.50 \pm 5.65 \mathrm{mmHg})$ and SO contraction period $(45.83 \pm 37.48$ to $19.00 \pm 29.46 \mathrm{~S})$. Three weeks after EST, the control group showed further reduced CBD pressure (0 $\mathrm{mmHg})$, SO pressure $(1.50 \pm 2.12 \mathrm{mmHg})$, SO contraction period ( $0 \mathrm{~S})$, SO contraction peaks (0) and SO contraction frequency $(0 / \mathrm{min})$, especially a significant reduction of $\mathrm{SO}$ contraction amplitude( $34.5 \pm 48.79$ to $0 \mathrm{mmHg}, \mathrm{P}=0.033)$. In contrast, the EEPP groups displayed SO function restoration, with all SOM parameters were comparable to those before the EST, including CBD pressure ( $11.5 \pm 7.31$ vs $11 \pm 2.16$ $\mathrm{mmHg})$, SO pressure $(17.50 \pm 17.75 \mathrm{vs} 18.20 \pm 21.39 \mathrm{mmHg})$, SO contraction amplitude $(53.67 \pm 21.54 \mathrm{vs}$ $60.00 \pm 36.08 \mathrm{mmHg})$, SO contraction period $(46.80 \pm 45.09$ vs $45.83 \pm 37.48 \mathrm{~S})$, SO contraction peaks $(4.20 \pm 3.27$ vs $4.17 \pm 4.26)$ and SO contraction frequency ( $8.16 \pm 10.88$ vs $3.05 \pm 3.29)$. These suggested that EST destroyed the function of SO, while EEPP restored SO function after EST.

\section{Discussion}

EST can damage the normal physiology of SO. The choledochoduodenal pressure gradient and SO basal pressure were totally lost 15 to 17 years after EST, following with duodenobiliary reflux[12]. The SO hypoactivity in terms of frequency and amplitude relates to gallstone formation[13]. In managing large 
CBD stones ( $>1 \mathrm{~cm}$ diameter), generous EST $(>1 \mathrm{~cm}$ ) usually was performed. In order to reduce the longterm risk of recurrent choledocholithiasis, endoscopic papillary balloon dilation (EPBD) or endoscopic papillary large balloon dilation (EPLBD) was an alternative option for large EST, recommended by European Society of Gastrointestinal Endoscopy guideline[14]. However, SO basal pressure was also dramatically reduced after EPBD, from $13.6 \mathrm{mmHg}$ to $6.3 \mathrm{mmHg}$ [12] and $9 \mathrm{mmHg}$ to $3.3 \mathrm{mmHg}$ [15] independently after 1 week. Even the SO basal pressure could recover to $9.3 \mathrm{mmHg}$ (one month) and 4.2 $\mathrm{mmHg}$ (one year) after EPBD, it was significantly lower than those before EPBD. For EPLBD, the SO basal pressure was reduced from $30.4 \mathrm{mmHg}$ to $6.4 \mathrm{mmHg}$ one week after procedure, similar result was observed one year after EPLBD [16]. Compared with EST, EPBD or EPLBD had similar CBD stones recurrent rates $[15,16]$.

Bile culture and proximal CBD biopsy indicated that bacteria colonization and biliary duct chronic inflammation after SO dysfunction [12]. In Korea $\$ 46,181$ patients with a history of CBD stones under endoscopic extraction were followed-up for an average 4.2 years, $11.3 \%(5,228 / 46,181)$ had the first CBD stone recurrence, $23.4 \%$ and $33.4 \%$ for the second and a third recurrence [17]. Although the exact etiology of gallstone formation was still not clear, bacteria probably play an important role in the formation of brown stones, which were usually found as recurrent CBD stones. By scanning electron microscopy and bile culture, Kaufman et. al. indicated that bacteria were only found in brown pigment stones. Furthermore, infected bile was found in $100 \%$ of those with recurrent choledocholithiasis[18]. Thus, how to preserve the SO function, reduce bacteria reflux and colonization in bile duct after CBD stone extraction need to be explored.

Inflammation, tissue formation and tissue remodeling were three phases of wound healing. The inflammatory phase involves recruitment of neutrophils, macrophages, inflammatory cells, chemotaxis and endothelial cells. Angiogenesis is a key player in the second proliferative phage, delayed or defective angiogenesis is implicated in healing impairment[19]. Local connective tissue fibroblasts at the wound edge are the major source of myofibroblasts, participated in tissue repair [20]. Compared with the EST control group, EEPP appeared to generate less neutrophils and macrophages infiltration, better angiogenesis and fibroblast proliferation. Based on the histological results, EEPP improved papillary healing after EST. Myofibroblasts is important in maintaining skin homeostasis and orchestrating tissue repair, which is characterized by expression of a-SMA [21]. Thus a-SMA expression is usually used as a marker of fibroblast differentiation, which is critical for tissue formation in the wound healing process. Half year after EEPP, a-SMA was highly expressed in the papilla wound surface, indicated a well healing process and scarring of papilla after EST. 
The SO is an important neuromuscular complex that located at the duodenum, and it controlled the volume of bile and pancreatic juice. It is believed that the long-term complications of EST are due to anatomic and physiologic disruption of SO. Up to now, SOM has been the best endoscopic method to identify the function of SO. Guelrud et al. have reported that the normal values for SOM as follows, CBD pressure was $6.8 \pm 1.7 \mathrm{~mm} \mathrm{Hg}$ with a range of $4-10 \mathrm{~mm} \mathrm{Hg}$, SO basal pressure $14.8 \pm 6.3 \mathrm{~mm} \mathrm{Hg}$ with a range of $4-30 \mathrm{~mm} \mathrm{Hg}$, SO amplitude $119.7 \pm 32.6 \mathrm{~mm} \mathrm{Hg}$ with a range of $76-180 \mathrm{~mm} \mathrm{Hg}$, SO frequency $5.7 \pm 1.2$ contractions/min with a range of $3-10$ contractions/min[22]. Similar results were obtained in both EST control and EEPP groups before any treatment. After EST, both SO basal pressure and CBD pressure dramatically reduced, but EEPP restored the SO pressure 3 weeks later.

For SO phasic contraction, amplitude, peak and period showed similar pattern with basal and CBD pressure. The decreasing SO myoelectric activity such as amplitude and frequency is related to the formation of gallstone formation[13]. Since EEPP restores SO contraction, EEPP may reduce the risk of recurrent choledocholithiasis. Moreover, SO contraction frequency even further increased in some animals. Whether EEPP can affect recurrent bile duct stone or other complications of EST, need to be further identified. Anatomically, pigs were different with human, with the biliary duct and pancreatic duct open separately at the duodenal bulb. Thus, post-EST pancreatitis was not observed in this study. However, due to small sample size and observation period limitations, whether the long-term complications of EST, especially recurrent choledocholithiasis could be reduced by EEPP need to be further investigated.

\section{Conclusions}

In conclusion, EEPP had accelerated and improved the papillary healing after EST, and preserved the SO physiological function. The importance of this report is that EEPP may be a safe and effective procedure for clinical use to reduce complications of EST.

\section{List Of Abbreviations}

endoscopic sphincterotomy, EST; endoscopic endoclip papilloplasty, EEPP; common bile duct, CBD; Sphincter of Oddi Manometry, SOM; smooth muscle actin, SMA; endoscopic papillary balloon dilation, EPBD; endoscopic papillary large balloon dilation, EPLBD.

\section{Declarations}

\section{Ethics approval and consent to participate}

All experimental procedures in this study were approved by the Ethical Committee of Laboratory Animals of Peking University Third Medical School. 


\section{Consent for publication}

Not Applicable.

\section{Availability of data and materials}

All data generated or analyzed during this study are included in this published article.

\section{Competing interests}

The authors declare that they have no competing interests.

\section{Funding}

This work was supported by the grants from The National Natural Science Foundation of China (81470905) and Capital Clinical Characteristic Application Research Project (Z181100001718149).

The funders support consumable items and expense needed in animal experiment procedures.

\section{Authors' contributions}

YW: acquisition or analysis and interpretation of the data, drafting the article, critical revision of the article for important intellectual content.

HC: study conception and design, critical revision of the article for important intellectual content.

YZ: study conception and design, acquisition and interpretation of the data.

KW: study conception and design, acquisition and interpretation of the data.

HZ: acquisition and interpretation of the data.

$\mathrm{XY}$ : study conception and design.

LM: acquisition and interpretation of the data.

WY: acquisition or analysis and interpretation of the data. 
$\mathrm{KL}$ : acquisition or analysis and interpretation of the data.

$\mathrm{YH}$ : guarantor of the article, study conception and design, critical revision of the article for important intellectual content.

All authors read and approved the final manuscript.

\section{Acknowledgements}

Not applicable.

\section{References}

1. Classen M, Demling L: Endoscopic sphincterotomy of the papilla of vater and extraction of stones from the choledochal duct (author's transl). Deutsche medizinische Wochenschrift (1946) 1974, 99(11):496-497.

2. Kawai K, Akasaka Y, Murakami K, Tada M, Koli Y: Endoscopic sphincterotomy of the ampulla of Vater. Gastrointestinal endoscopy 1974, 20(4):148-151.

3. Tanaka M, Takahata S, Konomi H, Matsunaga H, Yokohata K, Takeda T, Utsunomiya N, Ikeda S: Long-term consequence of endoscopic sphincterotomy for bile duct stones. Gastrointestinal endoscopy 1998, 48(5):465-469.

4. Kageoka M, Watanabe F, Maruyama Y, Nagata K, Ohata A, Noda Y, Miwa I, Ikeya K: Long-term prognosis of patients after endoscopic sphincterotomy for choledocholithiasis. Digestive endoscopy : official journal of the Japan Gastroenterological Endoscopy Society 2009, 21(3):170-175.

5. Oliveira-Cunha M, Dennison AR, Garcea G: Late Complications After Endoscopic Sphincterotomy. Surgical laparoscopy, endoscopy \& percutaneous techniques 2016, 26(1):1-5.

6. Nzenza TC, Al-Habbal Y, Guerra GR, Manolas S, Yong T, McQuillan T: Recurrent common bile duct stones as a late complication of endoscopic sphincterotomy. BMC gastroenterology 2018, 18(1):39.

7. Cai JS, Qiang S, Bao-Bing Y: Advances of recurrent risk factors and management of choledocholithiasis. Scandinavian journal of gastroenterology 2017, 52(1):34-43.

8. Afghani E, Lo SK, Covington PS, Cash BD, Pandol SJ: Sphincter of Oddi Function and Risk Factors for Dysfunction. Frontiers in nutrition 2017, 4:1.

9. Yang J, Jin H, Gu W, Zhang X, Zhang X: Determinants of long-term complications of endoscopic sphincterotomy are infections and high risk factors of bile duct and not sphincter of Oddi dysfunction. European journal of gastroenterology \& hepatology 2015, 27(4):412-418.

10. Fan X, Li X, Chang H, Yan X, Huang Y: Endoclip papilloplasty for a patulous and incompetent biliary papilla. VideoGIE : an official video journal of the American Society for Gastrointestinal Endoscopy 2019, 4(7):331-333. 
11. Huang $\mathrm{YH}$, Wang $\mathrm{K}$, Zhang HJ, Chang $\mathrm{H}$, Yan XE: A preliminary study of sphincter-preserving effect of SureClip from MicroTech on duodenal papilla occlusion. Chinese Jounal of Digestive Endoscopy. 2018; doi: 10.3760/cma.j.issn.1007-5232.2018.11.003.

12. Sato H, Kodama T, Takaaki J, Tatsumi Y, Maeda T, Fujita S, Fukui Y, Ogasawara H, Mitsufuji S: Endoscopic papillary balloon dilatation may preserve sphincter of Oddi function after common bile duct stone management: evaluation from the viewpoint of endoscopic manometry. Gut 1997, 41(4):541-544.

13. Rong ZH, Chen HY, Wang XX, Wang ZY, Xian GZ, Ma BZ, Qin CK, Zhang ZH: Effects of sphincter of Oddi motility on the formation of cholesterol gallstones. World journal of gastroenterology 2016, 22(24):5540-5547.

14. Kuo YT, Wang HP, Chang CY, Leung JW, Chen JH, Tsai MC, Liao WC: Comparable Long-term Outcomes of 1-Minute vs 5-Minute Endoscopic Papillary Balloon Dilation for Bile Duct Stones. Clinical gastroenterology and hepatology : the official clinical practice journal of the American Gastroenterological Association 2017, 15(11):1768-1775.

15. Yasuda I, Tomita E, Enya M, Kato T, Moriwaki H: Can endoscopic papillary balloon dilation really preserve sphincter of Oddi function? Gut 2001, 49(5):686-691.

16. Cheon YK, Lee TY, Kim SN, Shim CS: Impact of endoscopic papillary large-balloon dilation on sphincter of Oddi function: a prospective randomized study. Gastrointestinal endoscopy 2017, 85(4):782-790.e781.

17. Park BK, Seo JH, Jeon HH, Choi JW, Won SY, Cho YS, Lee CK, Park H, Kim DW: A nationwide population-based study of common bile duct stone recurrence after endoscopic stone removal in Korea. Journal of gastroenterology 2018, 53(5):670-678.

18. Kaufman HS, Magnuson TH, Lillemoe KD, Frasca P, Pitt HA: The role of bacteria in gallbladder and common duct stone formation. Annals of surgery 1989, 209(5):584-591; discussion 591-582.

19. Wietecha MS, DiPietro LA: Therapeutic Approaches to the Regulation of Wound Angiogenesis. Advances in wound care 2013, 2(3):81-86.

20. Higashiyama R, Nakao S, Shibusawa Y, Ishikawa O, Moro T, Mikami K, Fukumitsu H, Ueda Y, Minakawa $\mathrm{K}$, Tabata $Y$ et al: Differential contribution of dermal resident and bone marrow-derived cells to collagen production during wound healing and fibrogenesis in mice. The Journal of investigative dermatology 2011, 131(2):529-536.

21. Darby IA, Laverdet $B$, Bonté $F$, Desmoulière A: Fibroblasts and myofibroblasts in wound healing. Clinical, cosmetic and investigational dermatology 2014, 7:301-311.

22. Guelrud M, Mendoza S, Rossiter G, Villegas MI: Sphincter of Oddi manometry in healthy volunteers. Digestive diseases and sciences 1990, 35(1):38-46.

\section{Figures}


A
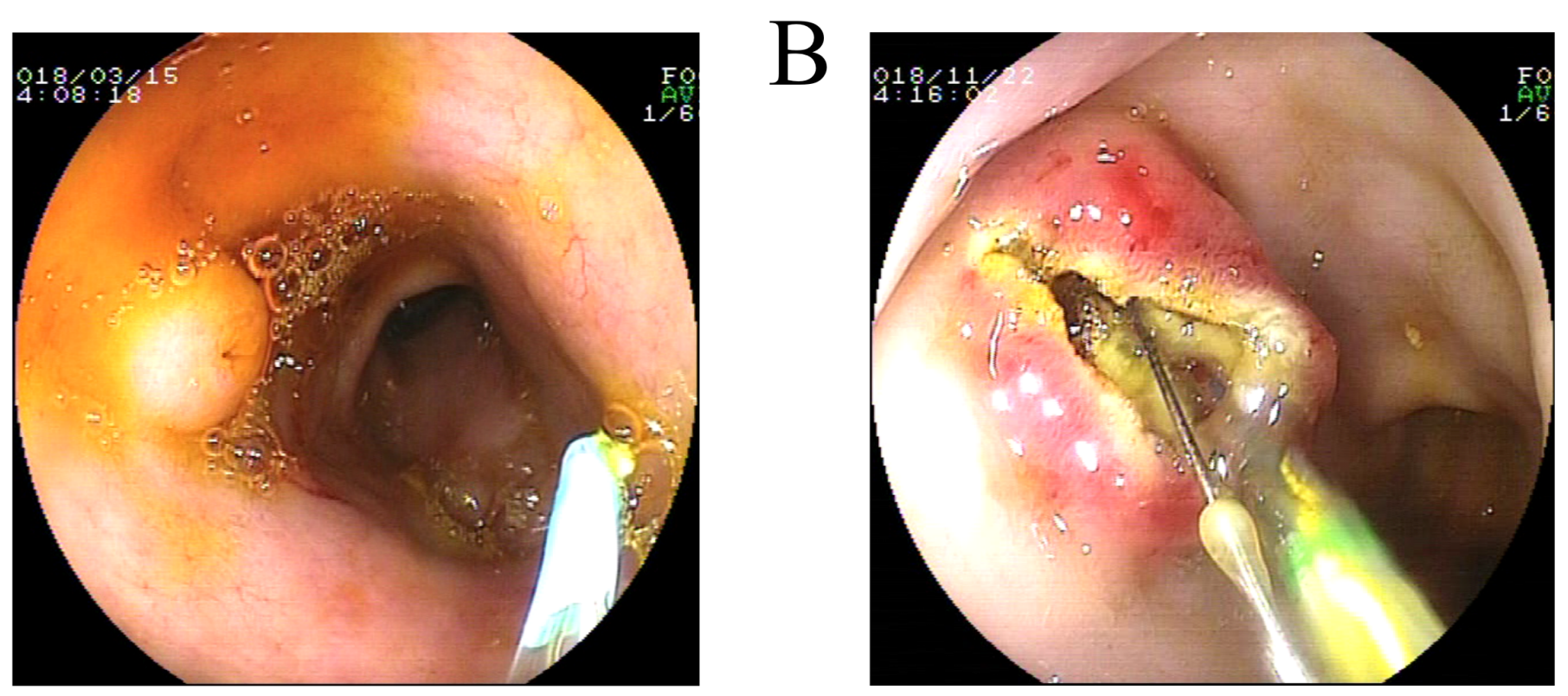

C
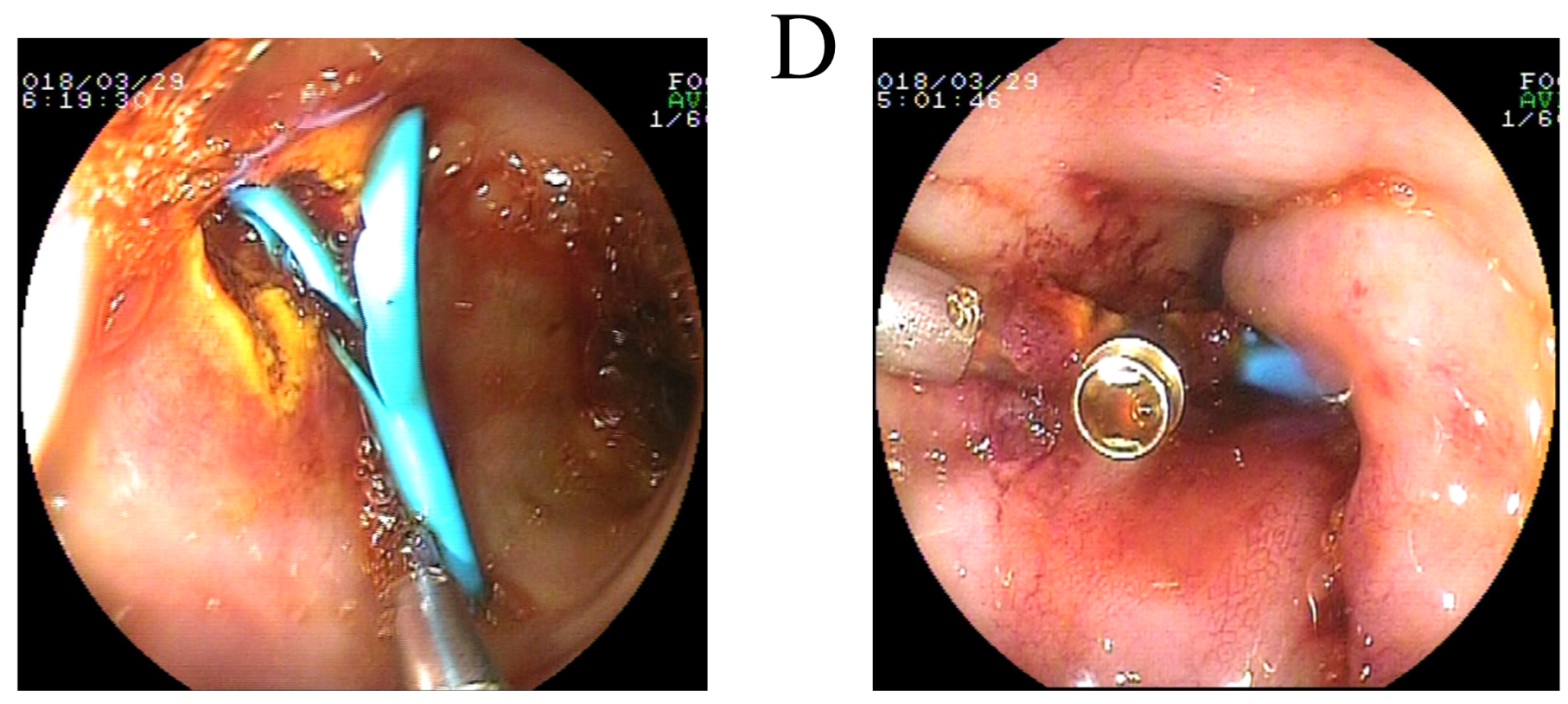

Figure 1

Endoclips endoscopic endoclip papilloplasty (EEPP) procedure. A) a untreated papilla. B) after a large biliary sphincterotomy $(>1 \mathrm{~cm})$. C) placement of $7 \mathrm{~F}$ plastic biliary stent. D) Zipper closure of the patulous papilla with MicroTech endoclips. 


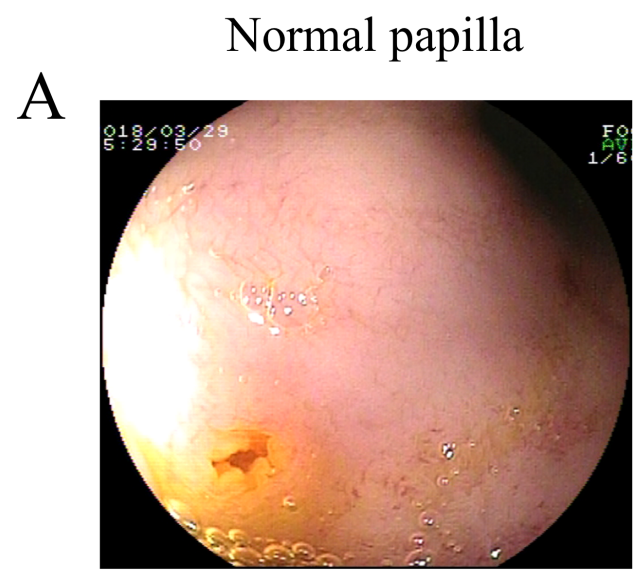

3 weeks after EST

B

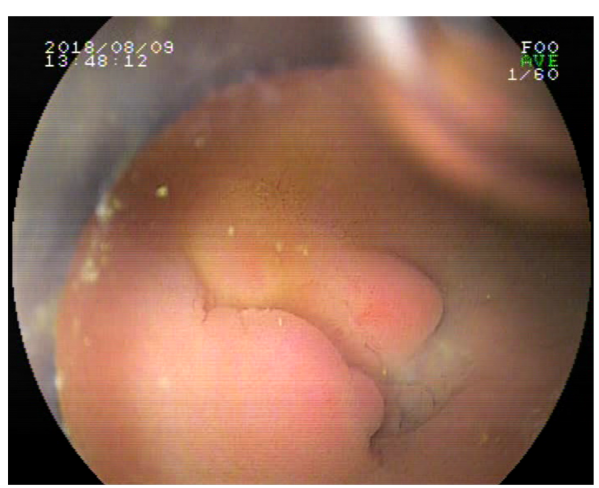

3 weeks after EEPP

C

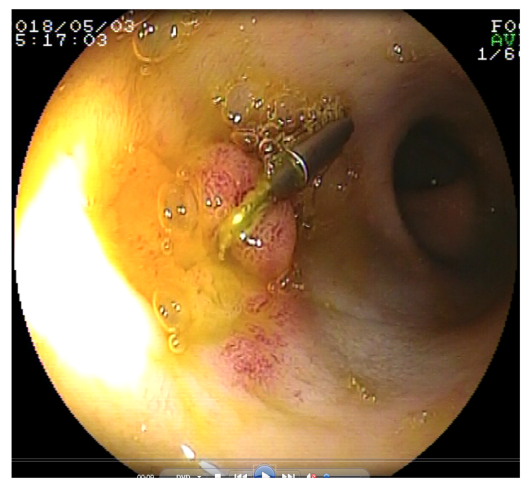

Figure 2

Endoscopic observation of the papilla. A) a untreated normal papilla. B) three weeks after endoscopic sphincterotomy. C) three weeks after endoscopic endoclip papilloplasty.

原

Figure 3

The histological changes of papillae, 3 weeks after EST/EEPP. A-C) 3 weeks after EST, hematoxylin-eosin staining of papilla. D-F) 3 weeks after EEPP, hematoxylin-eosin staining of papilla. Arrow indicates the wound after EST/EEPP. CBD, common bile duct. EST endoscopic sphincterotomy, EEPP, endoscopic endoclip papilloplasty. 


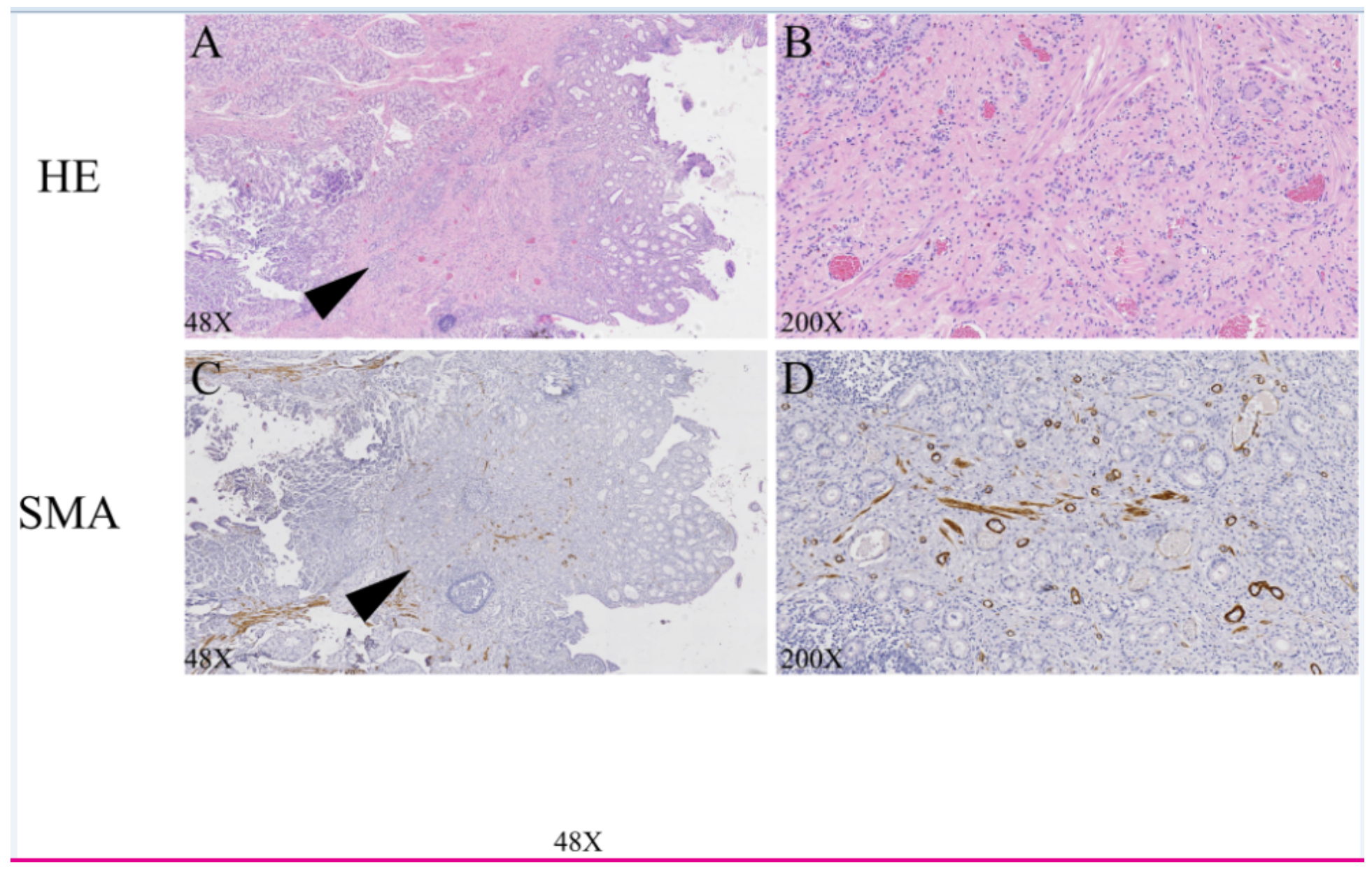

\section{Figure 4}

The histological changes of papilla, 24 weeks after EEPP. A-B) hematoxylin-eosin staining of papilla. C-D) immunohistochemistry staining of anti-a-SMA antibody. EEPP, endoscopic endoclip papilloplasty; a-SMA, smooth muscle actin. 
A
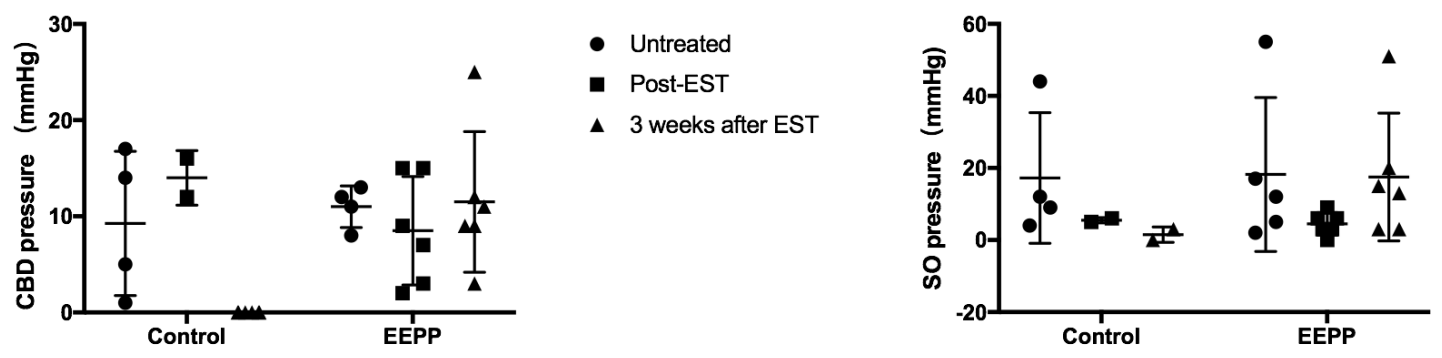

- Untreated

- post-EST

- Post-EST

- 3 weeks after EST

- 3 weeks after EST

C

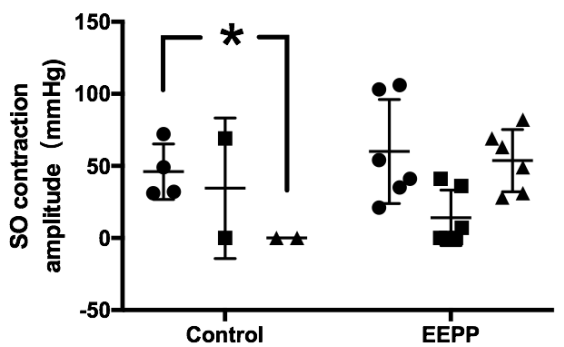

- Untreated

- post-EST

- 3 weeks after EST

D

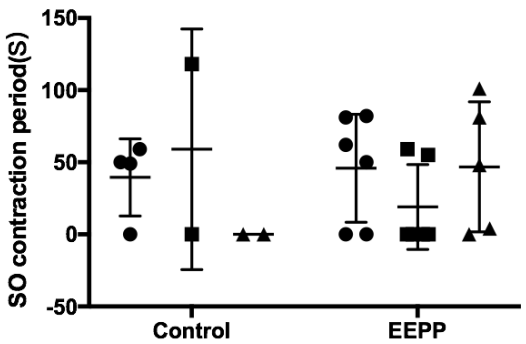

- Untreated

- Post-EST

- 3 weeks after EST

E

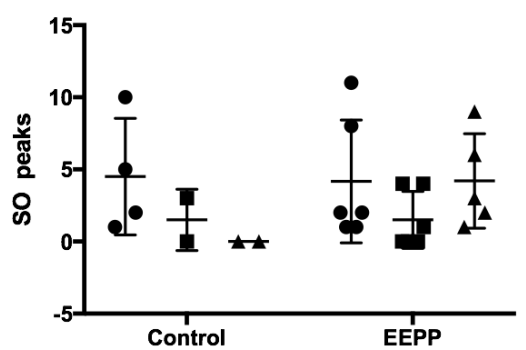

- Untreated

- Post-EST

\ 3 weeks after EST

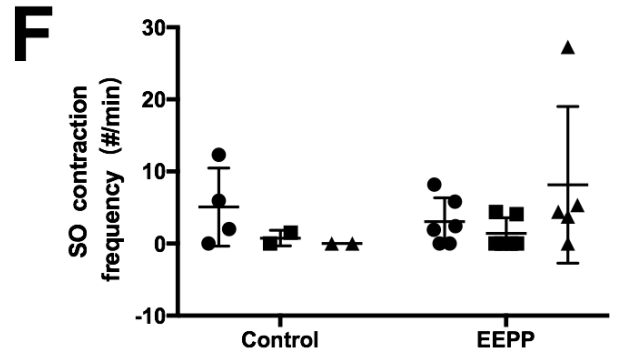

Figure 5

SOM results of control and EEPP groups at different time-points: at baseline, immediately after EST, and 3 weeks after procedure. A, CBD pressure. B, SO pressure. C, SO contraction amplitude. D, SO contraction period. E, SO contraction peaks. F. SO contraction frequency. SOM, sphincter of Oddi manometry; EEPP, endoscopic endoclip papilloplasty; EST endoscopic sphincterotomy; CBD, common bile duct; SO, sphincter of Oddi.

\section{Supplementary Files}

This is a list of supplementary files associated with this preprint. Click to download.

- NC3RsARRIVEGuidelinesChecklistfillableHYH.pdf 